\title{
Structure of the superconducting high-pressure phase of $\mathrm{Sc}_{3} \mathrm{CoC}_{4}$
}

\author{
Jan Langmann $\odot,{ }^{1}$ Marcel Vöst, ${ }^{1}$ Dominik Schmitz $\odot,{ }^{1}$ Christof Haas $\odot,{ }^{1}$ Georg Eickerling $\odot,{ }^{1, *}$ Anton Jesche, ${ }^{2}$ \\ Michael Nicklas $\odot{ }^{3}$ Arianna Lanza, ${ }^{4}$ Nicola Casati, ${ }^{5}$ Piero Macchi $\odot,{ }^{6}$ and Wolfgang Scherer ${ }^{1, \dagger}$ \\ ${ }^{1}$ CPM, Institut für Physik, Universität Augsburg, D-86159 Augsburg, Germany \\ ${ }^{2}$ Experimentalphysik VI, Zentrum für Elektronische Korrelation und Magnetismus, Institut für Physik, \\ Universität Augsburg, D-86159 Augsburg, Germany \\ ${ }^{3}$ Max Planck Institute for Chemical Physics of Solids, Nöthnitzer Straße 40, D-01087 Dresden, Germany \\ ${ }^{4}$ Center for Nanotechnology Innovation @ NEST, Istituto Italiano di Tecnologia, I-56127 Pisa, Italy \\ ${ }^{5}$ Swiss Light Source, Paul Scherrer Institut, CH-5232 Villigen, Switzerland \\ ${ }^{6}$ Dipartimento di Chimica, Materiali e Ingegneria Chimica “Giulio Natta,” Politecnico di Milano, I-20133 Milano, Italy
}

(Received 3 February 2021; revised 9 April 2021; accepted 20 April 2021; published 4 May 2021)

\begin{abstract}
We investigate pressure-induced structural changes to the Peierls-type distorted low-temperature phase of the low-dimensional $\mathrm{Sc}_{3} \mathrm{CoC}_{4}$ as a possible origin of its pressure-enhanced superconductivity. By means of cryogenic high-pressure x-ray diffraction experiments we could reveal subtle, but significant structural differences between the low-temperature phase at ambient and elevated pressures. We could thus establish the structure of the superconducting phase of the title compound, which interestingly still shows the main features of the Peierls-type distorted low-temperature phase. This indicates that in contrast to other low-dimensional materials a suppression of periodic structural distortions is no prerequisite for superconductivity in the transition-metal carbide.
\end{abstract}

DOI: 10.1103/PhysRevB.103.184101

\section{INTRODUCTION}

Structurally low-dimensional materials and dimensionality-driven physical effects are making their way into technical applications. Quantum dots [zero dimensional (0D)] are actively deployed in display technology $[1,2]$ or under intensive research for future uses in quantum computing [3]. Nanowires (1D) enable great improvements in photoand chemoelectric detectors and thermoelectric devices [4]. Transistors fabricated of atomically thin graphene layers (2D) might become an integral part of postsilicon microprocessors [5,6]. Furthermore, 3D superstructures of thin metal layers-arranged in the right way to break inversion symmetry-might provide promising candidates for diodes in superconducting electronics [7].

As indicated by the last example, the combination of structural low dimensionality with superconductivity can stimulate intriguing effects, even though Little's prediction of roomtemperature superconductivity in quasi-one-dimensional materials $[8,9]$ still remains an unobserved phenomenon at ambient pressure. Instead, a rich playing field of different ordering phenomena interacting with superconductivity has unfolded, e.g., structural transitions, charge-density and spin-density waves, and antiferromagnetism [10-20]. Superconducting compounds with intrinsically low-dimensional character have a special appeal to solid-state sciences, although the synthesis of large and defect-free single crystals is often challenging. This, for example, becomes evident from

\footnotetext{
*georg.eickerling@uni-a.de

${ }^{\dagger}$ wolfgang.scherer@uni-a.de
}

the large number of publications devoted to the well-known quasi-one-dimensional $\mathrm{NbSe}_{3}[13,14,21,22]$ or quasi-twodimensional graphite or graphene $[23,24]$ and transition-metal dichalcogenides [10,25-29].

The transition-metal carbide $\mathrm{Sc}_{3} \mathrm{CoC}_{4}$ crystallizes in a structure type combining quasi-1D and quasi-2D features. Quasi-1D $\left[\mathrm{Co}\left(\mathrm{C}_{2}\right)_{2}\right]_{\infty}$ ribbons extending along the crystallographic $b$ axis of the orthorhombic unit cell [Fig. 1(a)] are formed by covalent bonds between the cobalt atoms and $\mathrm{C}_{2}$ moieties [31]. Alternating stacking of the $\left[\mathrm{Co}\left(\mathrm{C}_{2}\right)_{2}\right]_{\infty}$ ribbons and scandium atoms ( $\mathrm{Sc} 1$ ) along the $a$ axis leads to quasi-2D layers [Fig. 1(b)]. Therein, neighboring $\left[\mathrm{Co}\left(\mathrm{C}_{2}\right)_{2}\right]_{\infty}$ ribbons with a separation of $3.39483(3) \AA$ are held together by subtle $\mathrm{Sc}-\mathrm{C}_{2}$ interactions. Additional scandium atom layers [ $\mathrm{Sc} 2$ and Sc3; Fig. 1(c)] are interleaved along the $c$ axis resulting in a large interlayer distance of $5.99855(5) \AA$ between adjacent Sc1-Co-C layers [30-37].

Superconductivity in $\mathrm{Sc}_{3} \mathrm{CoC}_{4}$ emerges below $T_{\mathrm{c}} \approx 4.5 \mathrm{~K}$ $[30,34,35]$ and is anticipated by a Peierls-type structural transition below $72 \mathrm{~K}$ [30]. Therein, the orthorhombic hightemperature (HT) phase structure (space group Immm) is transformed into the monoclinic low-temperature (LT) phase structure (space group $\mathrm{C} 2 / \mathrm{m}$ ) by a doubling of the translational period along the $\left[\mathrm{Co}\left(\mathrm{C}_{2}\right)_{2}\right]_{\infty}$ ribbons $[30,34,36,38]$. The exact degree and mode of interaction between this structural HT $\rightarrow$ LT phase transition and the onset of superconductivity at even lower temperatures are, however, not fully established yet. Furthermore, high-pressure studies of the electrical resistivity and magnetization in polycrystalline samples by Wang et al. [39] revealed a drastic increase in the superconducting volume at virtually constant $T_{\mathrm{c}}$ values. The authors rationalized this behavior by a pressure- and 
(a)
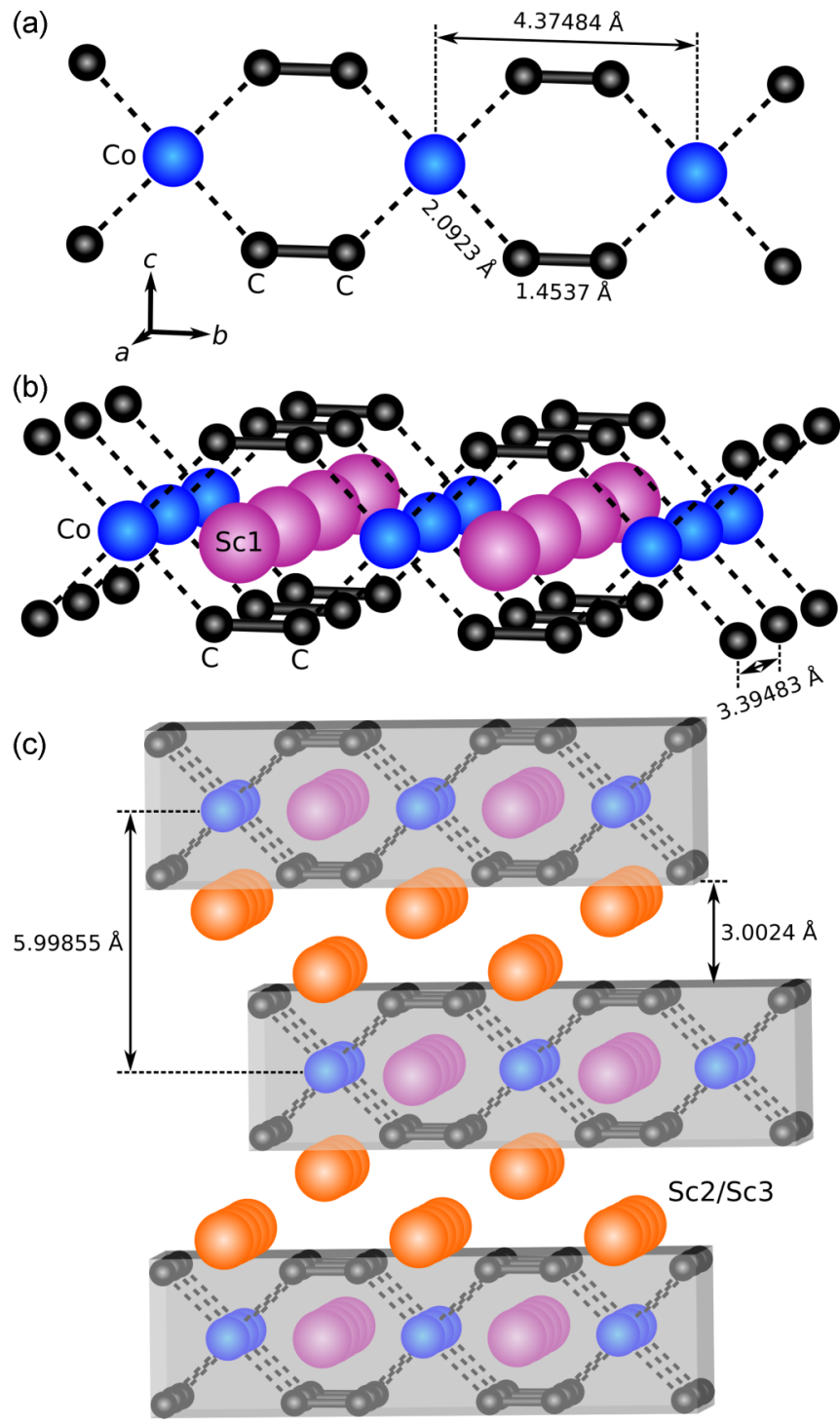

FIG. 1. (a) Infinite $\left[\mathrm{Co}\left(\mathrm{C}_{2}\right)_{2}\right]_{\infty}$ ribbon as basic quasi-1D building unit of the $\mathrm{Sc}_{3} \mathrm{CoC}_{4}$ structure; (b) composition of a quasi-2D layer by stacking $\left[\mathrm{Co}\left(\mathrm{C}_{2}\right)_{2}\right]_{\infty}$ ribbons and $\mathrm{Sc} 1$ atoms along the $a$ axis of the orthorhombic high-temperature phase; (c) decoupling of the quasi2D layers along the $c$ axis by interleaved $\mathrm{Sc} 2$ and $\mathrm{Sc} 3$ atoms. Salient interatomic distances are specified (structural data from Ref. [30]).

temperature-controlled coexistence of the HT and LT phase in the compound, whereby only the HT phase was supposed to become superconducting [39]. However, no structural information to verify this hypothesis has been provided up to now. Therefore we performed high-pressure and low-temperature single-crystal $\mathrm{x}$-ray diffraction studies in combination with physical property measurements to explore the pressureand temperature-dependent structure-property relationship in $\mathrm{Sc}_{3} \mathrm{CoC}_{4}$.

\section{METHODS}

Single- and polycrystalline samples of $\mathrm{Sc}_{3} \mathrm{CoC}_{4}$ were synthesized by arc-melting according to the method described in the literature $[31,37,40]$ and in addition from a lithium metal flux $[41,42]$ (full details of the synthesis and characterization methods employed can be found in the Supplemental Material [43]).

Magnetization measurements on a single-crystalline $\mathrm{Sc}_{3} \mathrm{CoC}_{4}$ sample were performed at various pressures up to $1.48 \mathrm{GPa}$ using a miniature ceramic anvil cell (mCAC) [44-48] and Daphne 7373 [49,50] as a pressure-transmitting medium. Applied pressures were determined at low temperatures by reference to the pressure dependence of $T_{\mathrm{c}}$ for an additional lead piece inside the pressure chamber [51,52]. Supplemental ambient-pressure measurements before and after the high-pressure study were performed by gluing the sample to a glass rod with GE Varnish. For all magnetization measurements a Quantum Design MPMS3 superconducting quantum interference device (SQUID) magnetometer was employed [43].

High-pressure electrical resistivity measurements up to $1.26 \mathrm{GPa}$ were performed employing a piston-cylinder-type pressure cell and silicon oil as pressure-transmitting medium. The single-crystalline $\mathrm{Sc}_{3} \mathrm{CoC}_{4}$ whisker was contacted by a four-point configuration using silver conductive paint and gold filaments. The pressure inside the pressure chamber was determined at low temperatures by measuring the $T_{\mathrm{c}}$ shift of a piece of lead [51,52]. For details of the setup, see Ref. [53]. The temperature-dependent resistivity measurements were carried out for various applied pressures upon cooling and heating cycles between 1.8 and $300 \mathrm{~K}$ in a Quantum Design PPMS using a Linear Research LR700 resistance bridge [54]. Additional ambient-pressure measurements of single-crystalline $\mathrm{Sc}_{3} \mathrm{CoC}_{4}$ whiskers four-point contacted with silver-epoxy resin were taken without surrounding pressure cell and using the standard dc-resistivity option of a Quantum Design PPMS. Uniaxial strain was created by gluing both ends of a whisker to a sapphire substrate using large droplets of silver-epoxy resin [43].

Pressure-dependent lattice parameters at room temperature were obtained from Le Bail fits [55,56] of synchrotron powder x-ray diffraction data with the software JANA2006 [57]. The respective diffraction experiments were carried out at the X04SA Materials Science (MS) beamline at the Swiss Light Source (SLS) [58,59] using a PSI Mythen II one-dimensional detector [60] and a membrane-driven diamond anvil cell (DAC). The pressure chamber was filled with finely ground and sieved $\mathrm{Sc}_{3} \mathrm{CoC}_{4}$ powder, and a 4:1 volume mixture of methanol and ethanol [61] was used as pressure-transmitting medium. $\alpha$-quartz powder was added for pressure calibration by reference to its well-known equation of state $[43,62]$.

The single-crystal $\mathrm{x}$-ray diffraction data in this paper were collected on a Huber four-circle Eulerian cradle goniometer equipped with a Dectris Pilatus CdTe $300 \mathrm{~K}$ pixel detector and an Incoatec $\mathrm{AgK}_{\alpha}$ microfocus sealed-tube $\mathrm{x}$ ray source $(\lambda=0.56087 \AA)$. Sample cooling to temperatures above $11 \mathrm{~K}$ was achieved utilizing an Advanced Research Systems closed-cycle helium cryocooler with exchangeable vacuum and radiation shields (beryllium or stainless steel with Kapton windows) [43].

High-pressure low-temperature $\mathrm{x}$-ray diffraction studies of $\mathrm{Sc}_{3} \mathrm{CoC}_{4}$ single crystals up to a pressure of $5.5 \mathrm{GPa}$ were carried out using a Diacell Tozer-type DAC $[63,64]$ (Almax easyLab) and Daphne 7575 as pressure-transmitting medium 

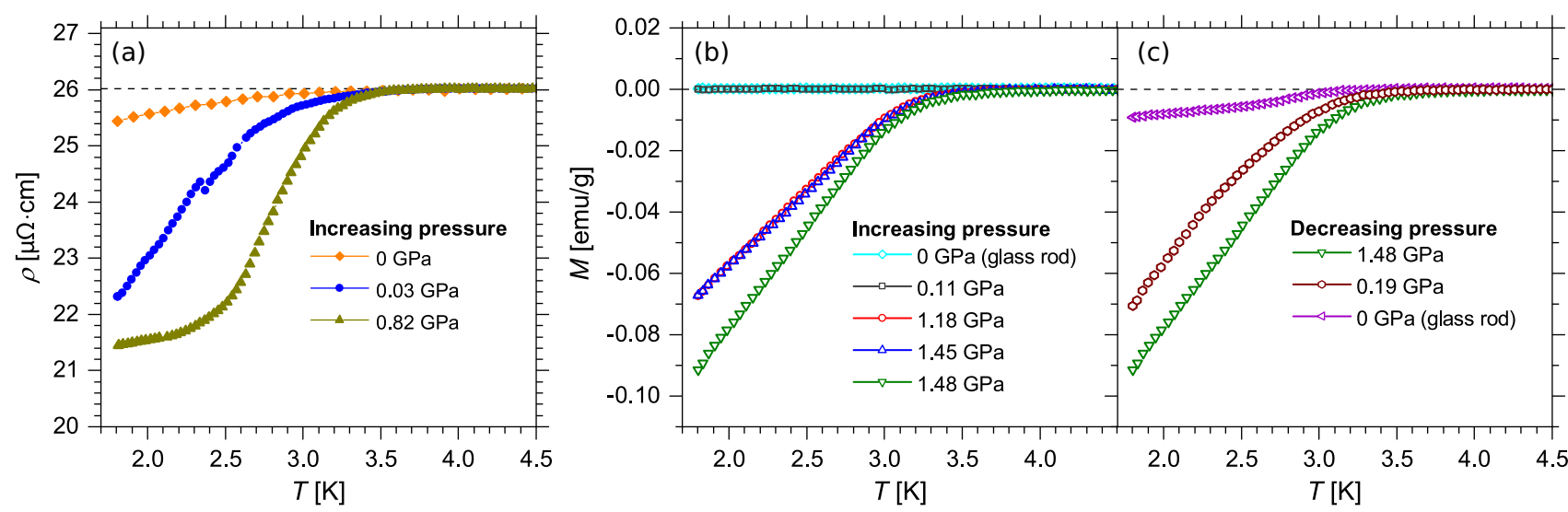

FIG. 2. Temperature- and pressure-dependent development of (a) the electrical resistivity $\rho(T)$ and (b) and (c) the magnetization $M(T)$ after zero-field cooling in a magnetic field of 5 Oe for the superconducting transition of $\mathrm{Sc}_{3} \mathrm{CoC}_{4}$. Data points in (a) and (b) were recorded while increasing the pressure, and data points in (c) were recorded while decreasing the pressure. Note that the ambient-pressure measurements of $\rho(T)$ (a) and $M(T)$ [(b) and (c)] were performed without a pressure cell. For better comparability, data points were brought to overlap at $4.5 \mathrm{~K}$ by applying shifts along the $\rho$ or $M$ axis.

[65]. Ruby spheres inside the pressure chamber allowed a pressure determination at room temperature via the ruby fluorescence method [66-68].

For high-pressure x-ray diffraction measurements on a $\mathrm{Sc}_{3} \mathrm{CoC}_{4}$ single crystal up to $10.1 \mathrm{GPa}$ at room temperature a Boehler-plate-type DAC [64,69] (Almax easyLab) was employed. The pressure determination method was analogous to the experiments with the Tozer-type DAC described above, but a 4:1 volume mixture of methanol and ethanol [61] was used as pressure-transmitting medium.

Obtained x-ray diffraction intensities were evaluated using the EVAL14 suite of programs [70,71] and subjected to scaling and absorption correction using the programs SADABS and TWINABS $[43,72]$. Structural refinements were performed with the program JANA2006 [57]. Resulting structural models in crystallographic information file (CIF) format may be obtained from Cambridge Crystallographic Data Centre (CCDC)/Leibniz Institute for Information Infrastructure (FIZ Karlsruhe) [73] or the Supplemental Material.

Density functional theory (DFT) calculations on the HT phase of $\mathrm{Sc}_{3} \mathrm{CoC}_{4}$ were performed employing the Vienna $a b$ initio simulation package (VASP) [74-77]. The Perdew-BurkeErnzerhof (PBE) density functional [78,79], an energy cutoff for the plane wave basis set of $500 \mathrm{eV}$, and a Brillouin grid sampling of $4 \times 4 \times 2$ were used throughout. The starting geometry for the ambient-pressure HT structure was adopted from the optimizations performed in Ref. [38], which are based on the same set of parameters.

Pressure-dependent geometry relaxations were performed at pressures of $2,4,6,8$, and $10 \mathrm{GPa}$. Optimizations were stopped when forces were smaller than $0.001 \mathrm{eV} / \AA$. Single-point self-consistent field (SCF) calculations enforcing uniaxial strain were performed by reducing the $a, b$, and $c$ lattice parameters of the relaxed HT ambient-pressure structure by \pm 0.02 and $\pm 0.04 \AA$.

All phonon dispersion calculations employing the finite displacement approach in a $2 \times 2 \times 2$ supercell were performed with the PHONOPY code [80] and VASP as force calculator using the same parameters as specified above.

\section{RESULTS AND DISCUSSION}

The starting point of our study involves the results published earlier by Wang et al. [39]. These authors found a significant increase in the superconducting volume fraction of polycrystalline $\mathrm{Sc}_{3} \mathrm{CoC}_{4}$ samples under the application of modest hydrostatic pressures. In the present study, we performed physical property measurements and $\mathrm{x}$-ray diffraction experiments on single-crystalline samples. This allows us to explore potential structure-property relationships in $\mathrm{Sc}_{3} \mathrm{CoC}_{4}$ and gain deeper insight into the origins of pressureenhanced superconductivity in the low-dimensional material. Also for single-crystalline samples a clear superconducting signature is only observed in the electrical resistivity $\rho(T)$ [see Fig. 2(a)] and the magnetization $M(T)$ [Figs. 2(b) and 2(c)] after application of pressure. It is noteworthy that the enhanced superconducting signal persists for several hours after decreasing the pressure from 1.48 to $0.19 \mathrm{GPa}$. It remains remanently present even after removing the sample from the pressure cell [see Fig. 2(c)]. This hints at a potential hysteretic behavior of the inherent structural changes induced by the application of pressure. Degradation of the sample quality as a possible origin of this behavior could be excluded by means of $\mathrm{x}$-ray diffraction before and after the high-pressure magnetization measurements [43].

In other low-dimensional compounds such as the transition-metal dichalcogenides $1 T-\mathrm{TiSe}_{2}, 2 \mathrm{H}-\mathrm{TaSe}_{2}$, and $2 \mathrm{H}-\mathrm{NbSe}_{2}$ the pressure-induced emergence of superconductivity is intimately linked to the suppression of a periodic structural distortion at low temperatures, i.e., a commensurate or incommensurate charge density wave [81-89]. We therefore tried to clarify whether the Peierls-type distortion leading to the LT phase $[30,38]$ might be suppressed upon application of pressure to enhance the superconductivity in $\mathrm{Sc}_{3} \mathrm{CoC}_{4}$ [39]. The structural properties of the ambient-pressure low-temperature phase have been studied earlier [30] and provide the starting point of this pressure- and temperature-dependent study. Atom displacements and bond lengths mentioned hereafter were determined in an ambient-pressure $\mathrm{x}$-ray diffraction experiment on a 

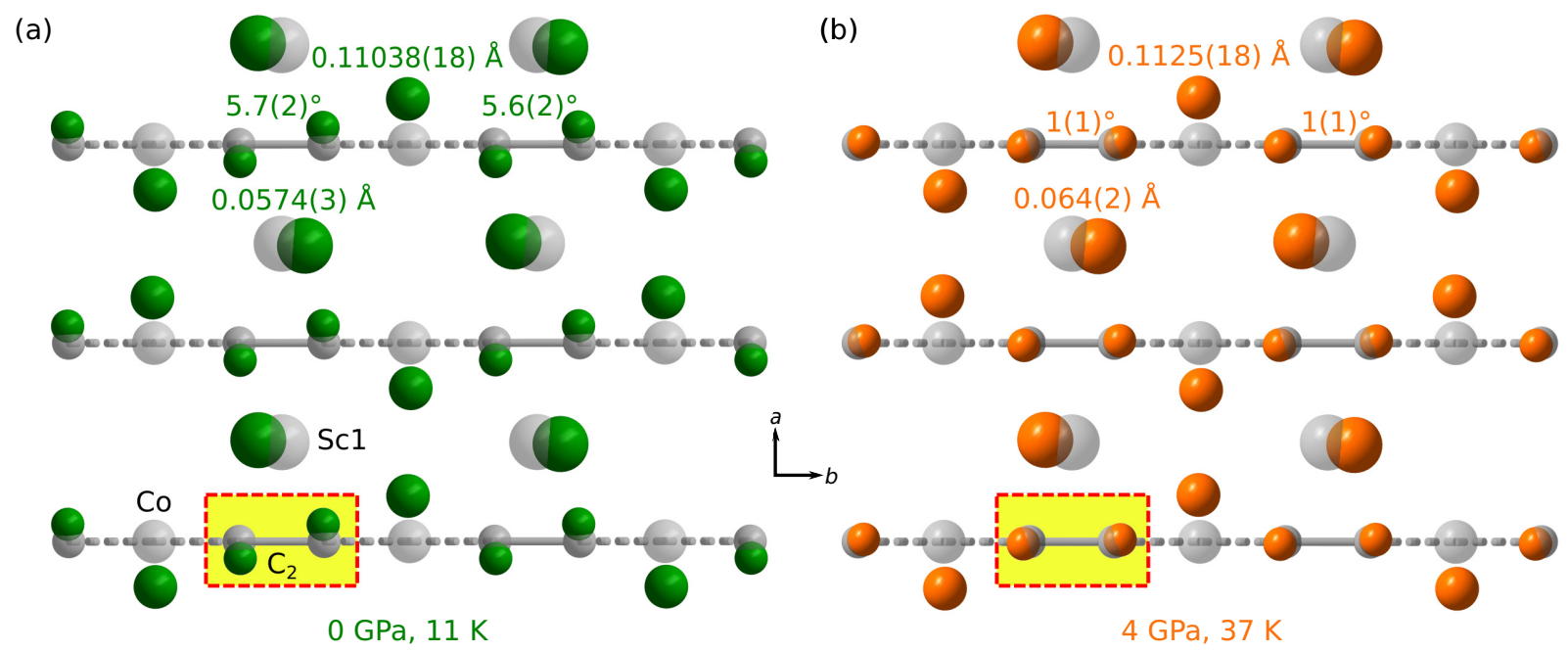

FIG. 3. Overlays of the refined atomic positions within a layered building unit of $\mathrm{Sc}_{3} \mathrm{CoC}_{4}$ at room temperature (gray, semitransparent; atomic positions from Ref. [30]) and after cooling to low temperatures (a) without or (b) with applied pressure (colored, nontransparent). All atom displacements are exaggerated sevenfold; Sc2 and Sc3 atoms have been omitted for clarity. Specified values of atom displacements and rotation angles are given with their threefold estimated standard deviation.

high-quality single-crystalline needle of $\mathrm{Sc}_{3} \mathrm{CoC}_{4}$ at $11 \mathrm{~K}$ (see Sec. II and the Supplemental Material [43] for further details). All bond lengths and displacements in this paper are given with their threefold standard deviation, while crystallographic directions are always specified with respect to the axes of the orthorhombic HT phase (space group Immm).

The LT phase of $\mathrm{Sc}_{3} \mathrm{CoC}_{4}$ (space group $C 2 / m$ ) is characterized by modulated displacements of $\mathrm{Co}, \mathrm{Sc} 1$, and $\mathrm{C}$ atoms from their HT phase positions in the quasi-2D layers of the $\mathrm{Sc}_{3} \mathrm{CoC}_{4}$ structure [see Fig. 3(a)]. Precisely, the cobalt atoms along a $\left[\mathrm{Co}\left(\mathrm{C}_{2}\right)_{2}\right]_{\infty}$ ribbon experience shifts of $\pm 0.11038(18) \AA$ relative to their crystallographic $2 d$ site in the HT phase (information on the calculation of the atom displacements is provided in the Supplemental Material [43]). Hence $\mathrm{Co}-\mathrm{Co}$ distances within chains of cobalt atoms along the $a$ axis display alternating larger [3.5985(9) $\AA$ ] and smaller [3.1569(6) $\AA]$ values compared with the constant separation of 3.3948(12) $\AA$ in the HT phase [30]. This modulation of the Co atomic positions is complemented by a modulation of the Sc1 atomic positions. Their displacements of \pm 0.0574 (3) $\AA$ with regard to the $2 b$ HT positions point along the $b$ axis and alternate along the $a$ axis; that is, their displacement direction is perpendicular to the modulation of the Co atomic positions. As can be seen in Fig. 3(a), the modulation of the Co and $\mathrm{Sc} 1$ atoms is correlated in such a way that the Sc1 atoms are shifted towards long $\mathrm{Co}-\mathrm{Co}$ contacts and evade short Co-Co contacts. In analogy to the arrangement of the cobalt atoms, this displacement pattern turns chains of equispaced scandium atoms along the $b$ axis [4.3748(12) $\AA$ ] above and below the $\left[\mathrm{Co}\left(\mathrm{C}_{2}\right)_{2}\right]_{\infty}$ ribbons into chains with alternating

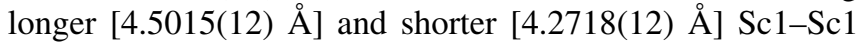
distances.

As a consequence of the $\mathrm{HT} \rightarrow \mathrm{LT}$ transition the $\left[\mathrm{Co}\left(\mathrm{C}_{2}\right)_{2}\right]_{\infty}$ ribbons are no longer planar, which is also reflected by rotations of the $\mathrm{C}_{2}$ units about rotation axes parallel to $c$. Due to the lack of a crystallographic $m$ plane perpendicular to $a$, rotations of adjacent $\mathrm{C}_{2}$ units about the $c$ axis in the same direction (conrotatory) or opposite directions (disrotatory) are both allowed by symmetry. The potential importance of the carbon atoms for superconductivity in $\mathrm{Sc}_{3} \mathrm{CoC}_{4}$ can be derived from isotopic substitution experiments: Replacement of ${ }^{12} \mathrm{C}$ by ${ }^{13} \mathrm{C}$ leads to a systematic suppression of the superconducting onset temperature $T_{c}^{\text {onset }}$ with an isotope coefficient $\alpha$ of 0.58 [90]. This observation is in line with the predictions of a DFT study by Zhang et al. [91]. The authors proposed that rotations of the $\mathrm{C}_{2}$ units and cobalt and scandium atom displacements are integral parts of key phonon modes coupling conduction electrons into superconducting Cooper pairs.

Yet, rotations of the $\mathrm{C}_{2}$ units are experimentally more difficult to assess by $\mathrm{x}$-ray diffraction than shifts of the heavy atoms cobalt and scandium. The latter displacements invariably lead to the appearance of prominent superstructure reflections with $k=\left(+\frac{1}{2},+\frac{1}{2}, 0\right)$ and also with $k^{\prime}=$ $\left(+\frac{1}{2},-\frac{1}{2}, 0\right)$ due to systematic pseudomerohedric twinning [see Fig. 6(f)] [30,38,40,92]. By contrast, carbon atom displacements may only contribute to the superstructure reflections in the case of disrotatory displacements of neighboring $\mathrm{C}_{2}$ units [93]. To obtain precise intensity information for main and superstructure reflections, we therefore employed long exposure times, a high-brilliance microfocus $\mathrm{x}$-ray source, and a noise-reduced pixel detector with high dynamic range in our single-crystal $\mathrm{x}$-ray diffraction experiments (see Sec. II and the Supplemental Material [43] for more details).

Our x-ray diffraction data hint at phase-pure LT-Sc ${ }_{3} \mathrm{CoC}_{4}$ at $11 \mathrm{~K}$ and ambient pressure without significant traces of the HT phase [43]. It can be fully described by an ordered structural model that is characterized by rotations of the two symmetry-independent $\mathrm{C}_{2}$ units in the same direction with rotation angles of $5.6(2)^{\circ}$ and $5.7(2)^{\circ}$ [highlighted in Fig. 3(a)]. Similar carbon atom shifts resulting in somewhat smaller but still conrotatory rotation angles of $2.8(4)^{\circ}$ and $3.0(4)^{\circ}$ have been found earlier at $9 \mathrm{~K}$ [30]. Notably, the 


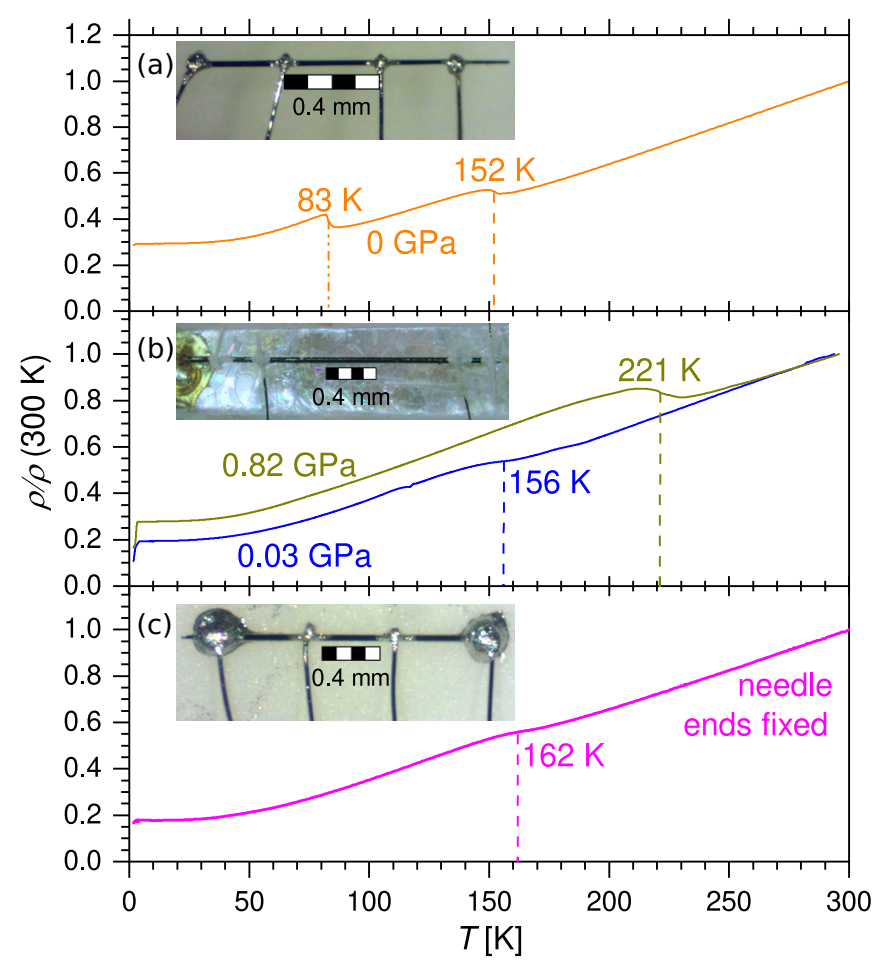

FIG. 4. Temperature-dependent electrical resistivity $\rho(T)$ of $\mathrm{Sc}_{3} \mathrm{CoC}_{4}$ single crystals (a) at ambient pressure and without fixation to a substrate, (b) at hydrostatic pressures of 0.03 and $0.82 \mathrm{GPa}$, and (c) glued at both ends on top of a sapphire chip. Insets: Photographic images of the respective samples after their preparation for measurements (a)-(c).

observed conrotatory displacements of subsequent $\mathrm{C}_{2}$ units along the $\left[\mathrm{Co}\left(\mathrm{C}_{2}\right)_{2}\right]_{\infty}$ ribbons result in the formation of shorter [2.098(4) $\AA$ ] and longer [2.113(3) $\AA$ ] Co-C distances in contrast to an alternative scenario with disrotatory displacements which would minimize all $\mathrm{Co}-\mathrm{C}$ distances. This rules out that strengthening of $\mathrm{Co}-\mathrm{C}$ bonds provides the only driving force of the carbon atom shifts in the LT phase structure.

In the next steps of our analysis we will aim at establishing possible pressure-dependent structure-property relationships to gain further insight into the potential origins of the characteristic superconducting behavior of $\mathrm{Sc}_{3} \mathrm{CoC}_{4}$. Measurements of the electrical resistivity $\rho(T)$ already hint towards potential pressure-induced modifications of our reference LT phase structure: As outlined earlier [38], the extended phonon softening process leading to a static Peierls-type structural distortion of $\mathrm{Sc}_{3} \mathrm{CoC}_{4}$ at ambient pressure is delimited by two pronounced anomalies in $\rho(T)$ at 152 and $83 \mathrm{~K}$ [Fig. 4(a)]. Our high-pressure experiments on single-crystalline samples in accordance with Wang et al. [39] show that only a single broad anomaly at $156 \mathrm{~K}$ can be observed in $\rho(T)$ after application of $0.03 \mathrm{GPa}$ [see blue curve in Fig. 4(b)]. This anomaly further shifts towards higher temperatures with increasing pressure [olive green curve in Fig. 4(b)]. A similar result, i.e., a suppression of the first anomaly at lower temperature in $\rho(T)$ and an upward shift of the second one by approximately $10 \mathrm{~K}$, can be obtained by fixing a single-crystalline $\mathrm{Sc}_{3} \mathrm{CoC}_{4}$ needle at two points along its long axis (i.e., parallel

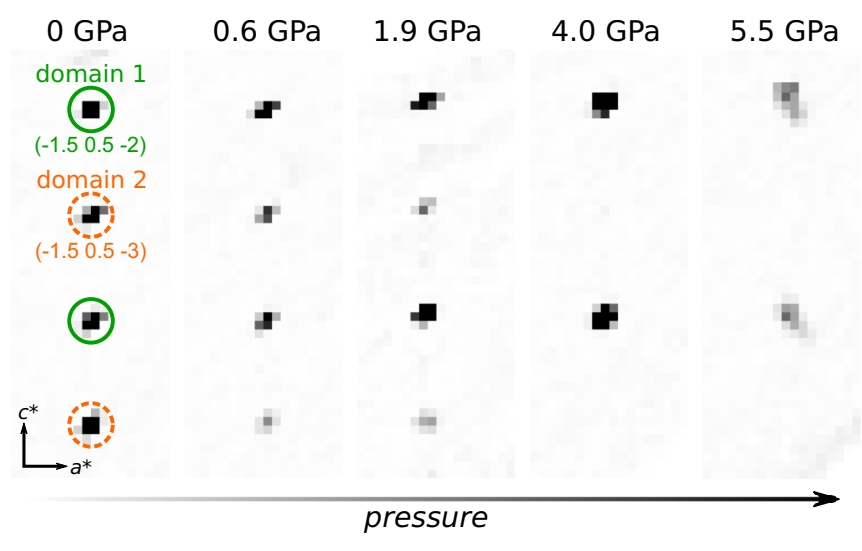

FIG. 5. Pressure-induced detwinning of a $\mathrm{Sc}_{3} \mathrm{CoC}_{4}$ sample at $T \approx 22 \mathrm{~K}$, as observed by $\mathrm{x}$-ray diffraction in the case of characteristic Bragg reflections from two twin domains (twin law [[-100], [010], [001]], i.e., an $m$ plane perpendicular to the $a$ axis of the orthorhombic HT unit cell). The displayed sections of the $(h, 0.5, l)$ plane contain only superstructure reflections and were recorded after applying pressures up to $5.5 \mathrm{GPa}$ and cooling to approximately $22 \mathrm{~K}$. The decreased scattering intensity at $5.5 \mathrm{GPa}$ is due to a beginning deterioration of the sample crystallinity.

to the crystallographic $a$ axis) on top of a sapphire chip [see Fig. 4(c)].

In order to investigate the origin of these pressuredependent changes in $\rho(T)$, we performed x-ray diffraction experiments at variable pressure and temperature. Inspection of Bragg intensities as well as atomic positions from structural refinements should reveal whether (i) only the distortion pattern during the HT $\rightarrow$ LT phase transition changes under pressure, (ii) the structurally distorted LT phase is suppressed in favor of the undistorted HT phase [39], or (iii) a designated and structurally distinct high-pressure LT phase of $\mathrm{Sc}_{3} \mathrm{CoC}_{4}$ is formed.

In support of hypothesis (i), our measurements indicate that the low-temperature superstructure Bragg reflections with $k=\left(+\frac{1}{2},+\frac{1}{2}, 0\right)$ can be observed up to pressures of $5.5 \mathrm{GPa}$. No indication of a pressure-induced structural phase transition connected with a change in the space group can be found. Instead, the collected diffraction data can still be described by a monoclinic lattice and comply with space group $C 2 / \mathrm{m}$. There is also no evidence for an incomplete phase transition under pressure leaving part of the atoms in their HT phase positions [43]. Differences between pressurized and unpressurized samples, however, may be recognized by comparing reconstructions of the $(h, 0.5, l)$ reciprocal space plane at $22 \mathrm{~K}$ for pressures between 0 and $5.5 \mathrm{GPa}$ (Fig. 5). Note that at ambient pressure, $\mathrm{Sc}_{3} \mathrm{CoC}_{4}$ crystals in the LT phase are systematically twinned with each of the two differently oriented twin domains contributing half of the superstructure reflections to the $(h, 0.5, l)$ plane (green and orange circles in Fig. 5). Cooling crystals below the HT $\rightarrow$ LT phase transition temperature at elevated pressures reveals that one-half of the superstructure reflections shows increasing intensities, whereas the other half shows decreasing intensities $(p \leqslant$ $1.9 \mathrm{GPa}$ ). The latter superstructure reflections finally vanish completely for $p>1.9 \mathrm{GPa}$, thus indicating a pressure- 


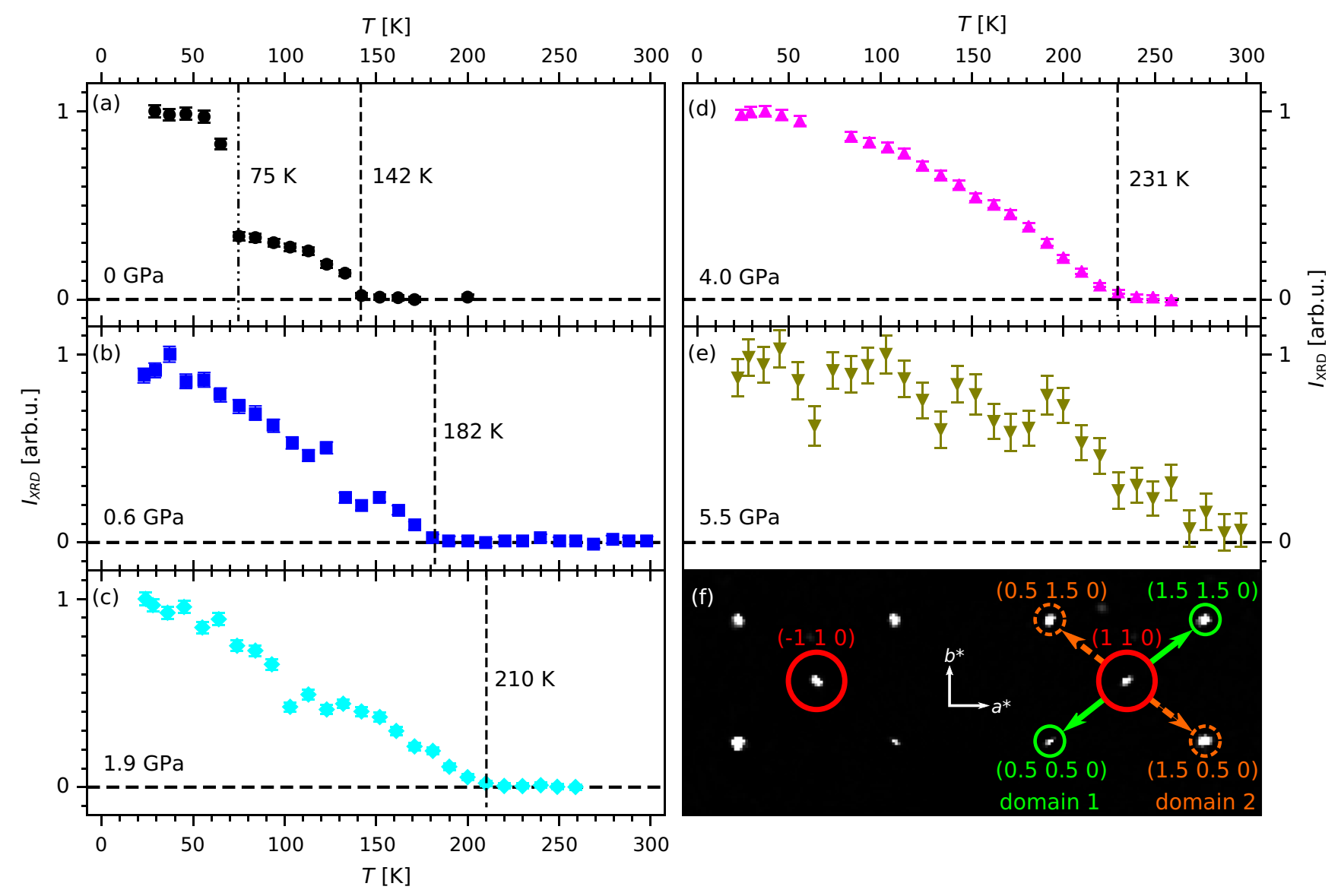

FIG. 6. Temperature dependence of the normalized integrated intensity $I_{\mathrm{XRD}}(T, p)$ with its threefold estimated standard deviation at the position of the $(-1.5,0.5,4)$ superstructure Bragg reflection for pressures between 0 and $5.5 \mathrm{GPa}(\mathrm{a})-(\mathrm{e})$. Note that some error bars are smaller than the employed data point symbols. A section of the ( $h k 0)$ plane (f) illustrates schematically the location of the superstructure reflections for twin domains 1 and 2 with respect to the main reflections.

induced detwinning of the crystal. Such a preference for one of the twin domains might originate from nonhydrostatic pressure conditions at the sample position inside the pressure cell [94].

Taking into account the otherwise unchanged characteristics of the LT phase in reciprocal space, we may connect the strongly modified behavior of the anomalies in $\rho(T)$ under pressure [compare Figs. 4(a) and 4(b)] to changes along the pathway from the HT to the LT phase. Therefore we determined the temperature and pressure dependence of the scattered intensity $I_{\mathrm{XRD}}(T, p)$ at the position of the $(-1.5,0.5,4)$ superstructure reflection (see Fig. 6). Consistent with $\rho(T)$, the increase in $I_{\mathrm{XRD}}(T, p)$ with decreasing temperature, which occurs in two steps at ambient pressure [Fig. 6(a)], renders into a continuous increase under pressure [Figs. 6(b)-6(e)]. We note, however, that a quantitative comparison of applied pressure values in $\rho(T)$ and $M(T)$ studies with those of the $\mathrm{x}$-ray diffraction experiments is hampered by the differing sample environments and pressure determination methods employed (see Sec. II and the Supplemental Material [43]).

From our data we may conclude that the energy difference between HT and LT phase is increased substantially, as is indicated by a shift in the onset temperature of the superstructure reflection intensity from $142 \mathrm{~K}$ at $0 \mathrm{GPa}$ [Fig. 6(a)] to $231 \mathrm{~K}$ at $4 \mathrm{GPa}$ [Fig. 6(d)]. A pressure of $5.5 \mathrm{GPa}$ preserves the superstructure reflections up to room temperature $\{$ Fig. 6(e), see also the Supplemental Material [43]\}, although at the cost of a degradation of the sample crystallinity. Interestingly, the isoelectronic and isostructural transition-metal carbides $\mathrm{Sc}_{3} \mathrm{IrC}_{4}$ and $\mathrm{Sc}_{3} \mathrm{RhC}_{4}$ also show a periodically distorted structure in analogy to the LT phase of $\mathrm{Sc}_{3} \mathrm{CoC}_{4}$ at room temperature but without prior cooling or pressure application $[95,96]$. There are, however, neither hints of superconductivity nor hints of the existence of an undistorted high-temperature phase comparable to $\mathrm{Sc}_{3} \mathrm{CoC}_{4}$ for these highly related compounds. In particular, systematic twinning as indicator of a potential $t_{2} \mathrm{HT} \rightarrow$ LT transition has not been observed in the iridium and rhodium congeners of $\mathrm{Sc}_{3} \mathrm{CoC}_{4}$ [96]. This discrepancy may be due to the fact that the $3 d$ metal cobalt is characterized by a significantly smaller covalent radius and weaker transition-metal-carbon bonds in comparison with the $4 d$ and $5 d$ group members rhodium and iridium. The resulting higher structural flexibility of $\mathrm{Sc}_{3} \mathrm{CoC}_{4}$ could thus be a prerequisite to allow the existence of both a HT and a LT phase structure.

The occurrence of a subtle competition between the HT and LT phases in $\mathrm{Sc}_{3} \mathrm{CoC}_{4}$ is reflected in the extended phonon softening regime preceding the $\mathrm{HT} \rightarrow \mathrm{LT}$ transition at ambient pressure [38]. Also its signatures in the temperature dependencies of $\rho(T)$ [Fig. 4(a)] and $I_{\mathrm{XRD}}(T, p)$ [Fig. 6(a)] 

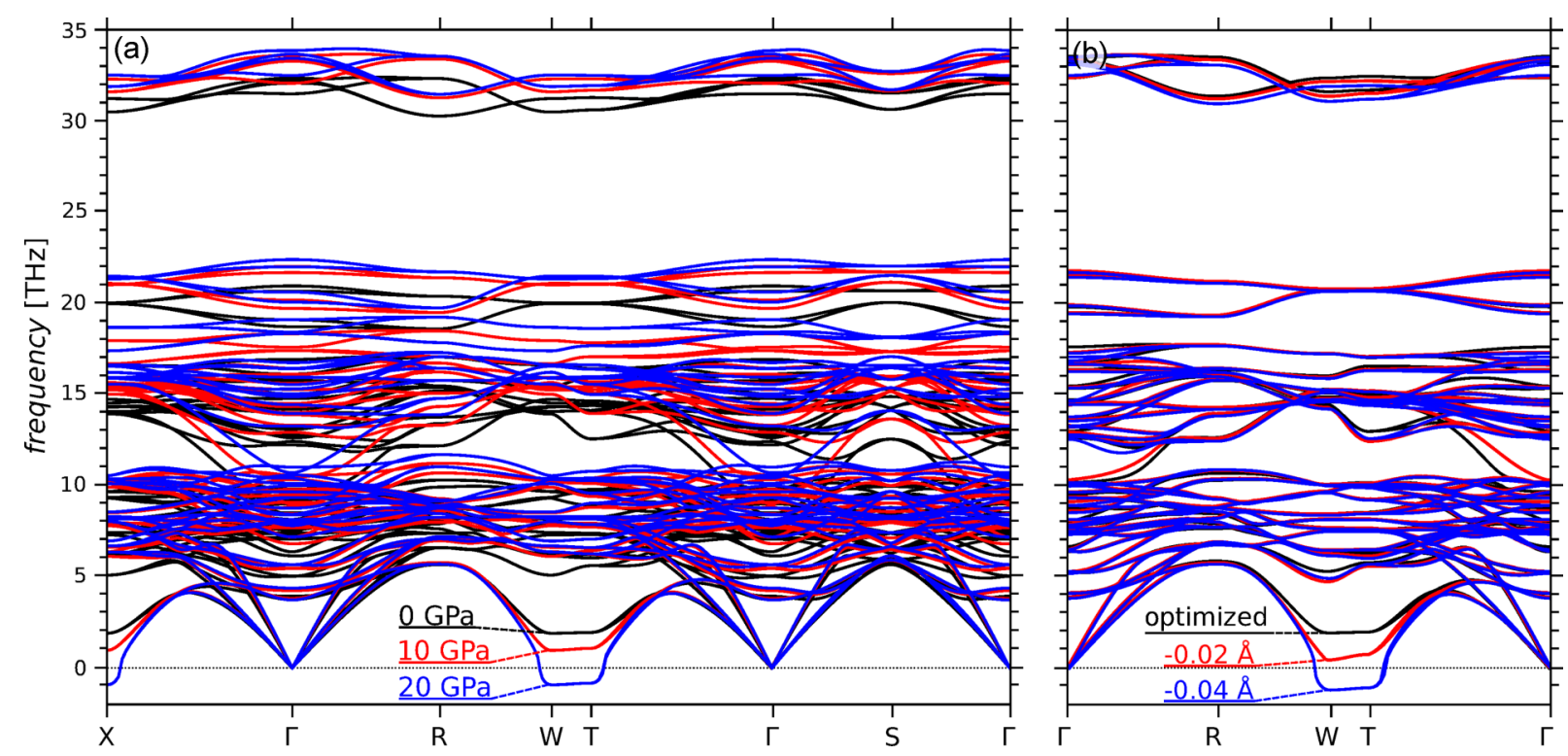

FIG. 7. Response of the phonon dispersion of $\mathrm{HT}-\mathrm{Sc}_{3} \mathrm{CoC}_{4}$ (DFT study) (a) to hydrostatic pressure and (b) to a reduction in the lattice parameter $a$.

react sensitively and in a highly related way already to small changes in pressure. Application of a pressure below 0.6 GPa is sufficient to induce a crossover of both physical properties from a course with two anomalies limiting the phonon softening regime towards low and high temperatures [38] to a course with a single anomaly [see Fig. 4(b) and Figs. 6(b)-6(e)]. Unfortunately, x-ray absorption by the employed pressure cell did not permit the investigation of the very weak and diffuse x-ray scattering features in analogy to Ref. [38]. Pressuredependent $a b$ initio phonon dispersion calculations for the HT phase structure, however, can provide more insight into the underlying causes for the modification of $\rho(T)$ and $I_{\mathrm{XRD}}(T, p)$ under pressure. Figure 7(a) illustrates the presence of a soft branch between the high-symmetry points $W$ and $T$ of the phonon dispersion already at ambient-pressure conditions. The phonon mode along the branch is characterized by displacements of the cobalt and scandium atoms $(\mathrm{Sc} 1)$ in the $a b$ plane anticipating their displacements in the LT phase of $\mathrm{Sc}_{3} \mathrm{CoC}_{4}$ [see Fig. 3(a)] [38]. On progressing along the path from $T$ to $W$ the LT-phase-like pattern of atom displacements at $T$ is modified by modulations of decreasing wavelength along the $c$ axis. Yet, carbon atom contributions to the mode in analogy to the displacements shown in Fig. 3(a) are absent. These can be found in a separate medium-frequency phonon mode at $\Gamma$ with still unclear behavior upon cooling below the HT $\rightarrow$ LT phase transition temperature. So far, there is only evidence for a profound temperature dependence of the $W-T$ phonon branch. Approaching the HT $\rightarrow$ LT transition temperature from above results in a reduction of the phonon frequency at $T$ to zero [38]. The same $W-T$ phonon branch also displays an extraordinary sensitivity to hydrostatic pressure \{see Fig. 7(a) and the Supplemental Material [43]\}. Its frequency is subjected to a strong and steady redshift with increasing pressure, while the frequencies of all other phonon branches show the expected blueshift. These trends indicate a gradual destabilization of the HT phase structure with increasing pressure in line with the experimentally observed pressure-induced enhancement of the transition temperature from the HT to the LT phase [97].

Despite the red frequency shift of the $W-T$ phonon branch in our calculations [Fig. 7(a)] and the preservation of the low-temperature superstructure reflections upon heating to room temperature at $5.5 \mathrm{GPa}$ in our diffraction experiments [Fig. 6(e)], application of pressure alone does not suffice to induce a transition of $\mathrm{Sc}_{3} \mathrm{CoC}_{4}$ from the HT to the LT phase structure. No superstructure reflections could be observed in single-crystal XRD experiments up to the destruction of the sample at $10.1 \mathrm{GPa}$, when the pressure cell was kept constantly at room temperature [43]. An overlay of structural models at 0.2 and $4.2 \mathrm{GPa}$ in the Supplemental Material [43] illustrates that the pressure-induced shifts of the atomic positions remain negligible under these conditions. Likewise, no phase transition could be inferred from the pressure dependence of the lattice parameters obtained from room-temperature powder XRD experiments [Fig. 8(a)]. The experimentally observed linear decrease in the lattice parameters upon application of up to $10.1 \mathrm{GPa}$ with a stronger absolute compression of $c(\Delta c=0.20 \AA)$ compared with $a$ and $b(\Delta a=0.07 \AA, \Delta b=0.06 \AA)$ is very well reproduced by DFT studies of the compressibility behavior of the HT phase [Fig. 8(a)].

This behavior might be related to the strongly differing development of the lattice parameters of $\mathrm{Sc}_{3} \mathrm{CoC}_{4}$ either upon cooling or upon increasing hydrostatic pressure. Previous variable-temperature neutron diffraction studies between 277 and $1.8 \mathrm{~K}$ showed that a reduction in temperature is accompanied by increases in the $b$ and $c$ parameters by approximately $0.01 \AA$ and a decrease in the $a$ parameter by approximately $0.02 \AA$ [Fig. 8(b)] [30]. An increase in hydrostatic pressure, by contrast, results in the compression of all lattice parameters. Thus a strongly anisotropic pressure response of $\mathrm{Sc}_{3} \mathrm{CoC}_{4}$ may be suspected in accordance with the low-dimensional structure of the compound. The validity of this hypothesis is underlined by the fact that the application of uniaxial stress 

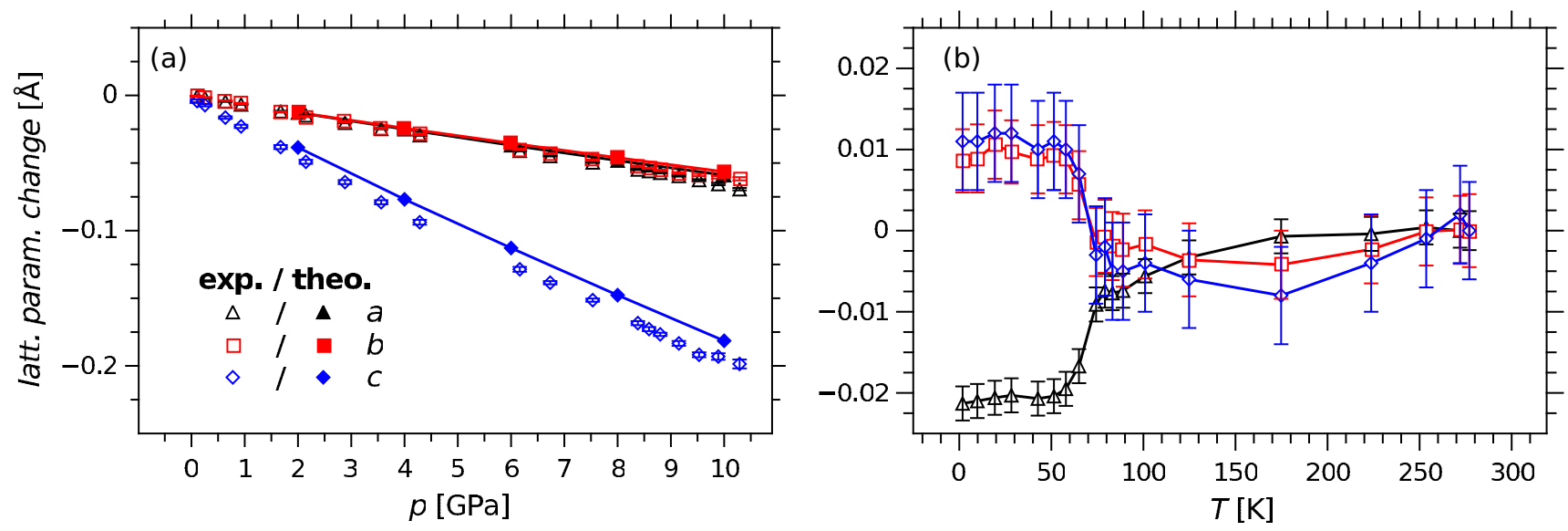

FIG. 8. Experimentally (exp., open symbols) and theoretically derived (theo., solid symbols) changes in the lattice parameters (latt. param.) of $\mathrm{Sc}_{3} \mathrm{CoC}_{4}$ (a) with varying pressure at constant temperature and (b) with varying temperature at ambient pressure (data from Ref. [30]). Error bars indicate the threefold estimated standard deviation of the experimental data (some error bars are smaller than the employed symbols).

along the long axis of single-crystalline $\mathrm{Sc}_{3} \mathrm{CoC}_{4}$ needles leads to pronounced changes in the temperature-dependent electrical resistivity [see Fig. 4(c)]. We therefore performed a DFT study probing the response of the phonon dispersion to a uniaxial compression along each of the three unit cell axes by varying the HT phase lattice parameters independently [43]. The strongest frequency decrease along the $W-T$ phonon branch is obtained by a reduction in the $a$ parameter correlating with the distance between adjacent $\left[\mathrm{Co}\left(\mathrm{C}_{2}\right)_{2}\right]_{\infty}$ ribbons [see red and blue curves in Fig. 7(b)]. Negative frequencies along the path indicate the instability of the HT phase structure after a compression of the $a$ lattice parameter by more than approximately $0.02 \AA$. A similar dispersion behavior is only achieved by the application of hydrostatic pressure in the range of $20 \mathrm{GPa}$.

After pointing out the energetic destabilization of the HT phase under pressure we will now focus on the pressureinduced structural changes to the LT phase. Although the space group $C 2 / m$ applies to the LT phase structure under ambient-pressure and high-pressure conditions, some degrees of freedom for the atom arrangement remain. We find, for example, that the distance between adjacent $\left[\mathrm{Co}\left(\mathrm{C}_{2}\right)_{2}\right]_{\infty}$ ribbons is reduced from 3.378(7) $\AA$ at 0 GPa to 3.34(2) $\AA$ at 4 GPa. An even smaller compression from 6.0105(3) $\AA$ to 5.9849(8) $\AA$ is found for the interlayer distance between adjacent quasi-2D Sc1-Co-C layers. Further free parameters in the HT $\rightarrow$ LT phase transition involve the magnitude of the cobalt and scandium atom shifts, and the extent and relative orientation of the $\mathrm{C}_{2}$-unit rotations. Figures 3 (a) and $3(\mathrm{~b})$ visualize the effect on the atom positions, when $\mathrm{Sc}_{3} \mathrm{CoC}_{4}$ is cooled to $T<40 \mathrm{~K}$ with and without an applied pressure of $4 \mathrm{GPa}$, respectively.

It becomes evident that the general displacement pattern of the cobalt and scandium atoms in the LT phase remains rather invariant upon pressure application: At both 0 and $4 \mathrm{GPa}$, the cobalt atoms along the $\left[\mathrm{Co}\left(\mathrm{C}_{2}\right)_{2}\right]_{\infty}$ ribbons are shifted in the positive and negative $a$ direction by similar extents of $0.11038(18) \AA$ and $0.1125(18) \AA$, respectively. Also the shifts of the $\mathrm{Sc} 1$ atoms along the $b$ axis are remarkably similar with values of $0.0574(3) \AA$ at $0 \mathrm{GPa}$ and $0.064(2) \AA$ at $4 \mathrm{GPa}$ and $37 \mathrm{~K}$. By contrast, the rotation angles of the $\mathrm{C}_{2}$ units obtained from the structural refinements display a distinct pressure dependency [highlighted in Figs. 3(a) and 3(b)]: At $0 \mathrm{GPa}$ and $11 \mathrm{~K}$, the $\mathrm{C}_{2}$ units are subjected to significant rotations out of their HT phase positions with rotation angles between $5.6(2)^{\circ}$ and $5.7(2)^{\circ}$. Nearly vanishing rotation angles of $1(1)^{\circ}$ are, however, observed at $4 \mathrm{GPa}$ and $37 \mathrm{~K}$ [43]. This observation might be linked to the pressure-induced detwinning of $\mathrm{Sc}_{3} \mathrm{CoC}_{4}$ samples: The two possible twin domains in the ambient-pressure LT phase mainly differ by a different rotation sense of the $C_{2}$ units in an otherwise nearly unchanged arrangement of cobalt and scandium atoms. A vanishing $C_{2}$ rotation angle makes these twin domains nearly identical leaving only marginal differences in the cobalt and scandium atom positions [43]. As a result, the realization of a single-domain state extending over the entire sample volume might be favored. The absence of further anomalies in the electrical resistivity between $37 \mathrm{~K}$ and the superconducting transition temperature at $4.5 \mathrm{~K}$ [Fig. 4(b)] implies that this detwinned high-pressure low-temperature phase is the one hosting superconductivity in $\mathrm{Sc}_{3} \mathrm{CoC}_{4}$.

In a last step we proceed to link the observed temperatureand pressure-dependent changes in the superstructure reflection intensity $I_{\mathrm{XRD}}(T, p)$ (see Fig. 6) to changes observed in the atomic positions. To do so, we performed x-ray diffraction experiments at 0 and $4 \mathrm{GPa}$ for selected temperatures above and below the steplike increase in $I_{\mathrm{XRD}}(T, p)$ observed at approximately $80 \mathrm{~K}$ and $0 \mathrm{GPa}$. Figure 9 gives an overview of the observed temperature dependence of the Co (blue circles) and $\mathrm{Sc} 1$ atom displacements (magenta rectangles) and the $\mathrm{C}_{2}$-unit rotations (black triangles) at $0 \mathrm{GPa}$ [Fig. 9(a)] and $4 \mathrm{GPa}$ [Fig. 9(b)]. Data points from x-ray diffraction experiments with and without usage of a DAC are indicated by solid and open symbols, respectively.

It turns out that the structural models obtained at ambient pressure and above $75 \mathrm{~K}$ represent intermediate steps in the progression of $\mathrm{Sc}_{3} \mathrm{CoC}_{4}$ towards its final state below $75 \mathrm{~K}$ [see Fig. 9(a)]. Consistent with the nonzero value of the superstructure reflection intensity in the same temperature and pressure regime [Fig. 6(a)], shifts of the atoms from their positions in the HT phase structure are already 


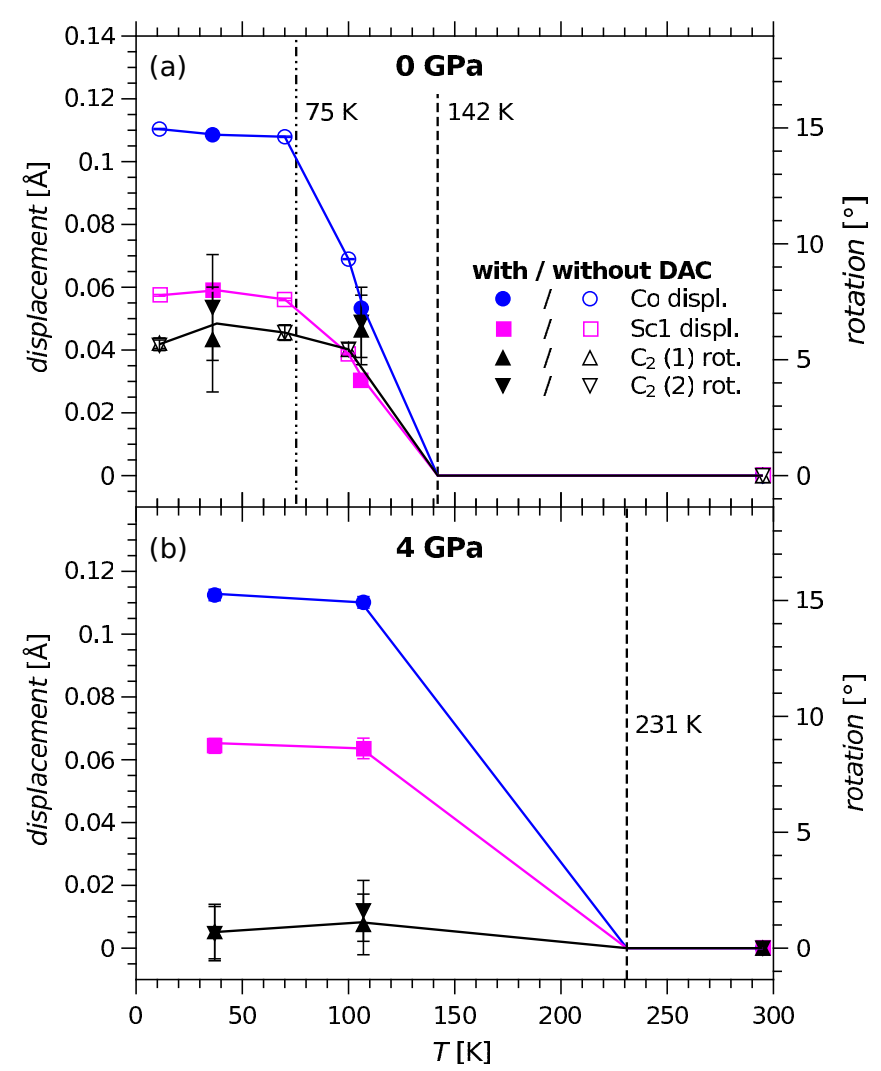

FIG. 9. Temperature dependence of the displacements (displ.) of the Co (blue circles) and Sc1 atoms (magenta squares) and the rotation angles (rot.) of the two symmetry-inequivalent $\mathrm{C}_{2}$ units $\left[\mathrm{C}_{2}\right.$ (1) and $\mathrm{C}_{2}$ (2), black triangles] for (a) $0 \mathrm{GPa}$ and (b) $4 \mathrm{GPa}$. Carbon atom displacements corresponding to each $\mathrm{C}_{2}$ rotation angle (right ordinate) are specified on the left ordinate. Note that error bars indicate the threefold estimated standard deviation of all data points (some error bars are smaller than the employed symbols). Vertical lines denote critical temperatures determined from the intensity of an individual superstructure reflection (Fig. 6). Thereby, dashed lines refer to the onset of intensity at its position, and a dash-dotted line marks the additional jumplike increase in intensity observed at $0 \mathrm{GPa}$. Solid lines connecting the data points serve as guides to the eye.

present at this stage [Fig. 9(a)]. Thereby, larger values of $I_{\mathrm{XRD}}(T, p)$ correspond to larger shifts of the atomic positions. It should be emphasized, however, that the phonon softening process during the $\mathrm{HT} \rightarrow \mathrm{LT}$ transition is not yet completed for temperatures above $75 \mathrm{~K}$, so that all atom displacements are likely to be of a correlated, but still dynamical nature $[38,43]$. When the atom displacements become rather static on cooling below $75 \mathrm{~K}$, a steplike increase and a subsequent saturation of the superstructure reflection intensity are observed.

As already stated above, direction and maximal displacements of the Co and $\mathrm{Sc} 1$ atoms are not altered significantly by applying a pressure of $4 \mathrm{GPa}$. Yet, the absence of a step in $I_{\mathrm{XRD}}(T, p)$ and the higher onset temperature of $231 \mathrm{~K}(0$ GPa: $142 \mathrm{~K}$ ) correlate with the attainment of these maximum displacements at temperatures significantly above $75 \mathrm{~K}$ [see Fig. 9(b)]. The main structural difference between 0 and $4 \mathrm{GPa}$ can thus be attributed to the different extent of the out-ofplane rotation of the $\mathrm{C}_{2}$ units at all investigated temperatures. Hence the application of pressure effectively prevents the displacement of the carbon atoms from their HT phase positions but does not suppress the formation of a periodic structural distortion.

\section{CONCLUSION}

To conclude, we suggest a connection between the occurrence of volume superconductivity and subtle structural modifications to the known Peierls-type distorted low-temperature (LT) phase of $\mathrm{Sc}_{3} \mathrm{CoC}_{4}[30,38,39]$ under pressure. We demonstrated that the compound forms ordered LT phase structures under both ambient- and elevated-pressure conditions without remaining HT phase contributions. Differences between the ambient- and elevated-pressure LT phase structures are limited to a reduction in the $\mathrm{C}_{2}$ rotations out of the $\left[\mathrm{Co}\left(\mathrm{C}_{2}\right)_{2}\right]_{\infty}$ ribbon plane from $5.6^{\circ}-5.7^{\circ}$ to nearly zero. This brings the $\mathrm{C}_{2}$ moieties back to their HT phase positions in an otherwise still distorted arrangement of cobalt and scandium atoms.

On an atomistic level the changed equilibrium positions of the $\mathrm{C}_{2}$ units may affect phononic and electronic properties of the electron-phonon coupling driven superconductor $\mathrm{Sc}_{3} \mathrm{CoC}_{4}$. The importance of carbon atom vibrations for the emergence of superconductivity is highlighted by ${ }^{12} \mathrm{C} /{ }^{13} \mathrm{C}$ isotopic substitution experiments indicating a clearly nonzero isotope coefficient of 0.58 [90]. A key role of $\mathrm{C}_{2}$ librational modes in the coupling of conduction electrons into Cooper pairs is also pointed out by DFT studies employing the Eliashberg formalism [91]. Thus establishing structureproperty relationships in favor of pressure-induced volume superconductivity presents an interesting, but challenging task for future theoretical studies.

However, the subtle changes in the $\mathrm{C}_{2}$ rotation also affect the properties of $\mathrm{Sc}_{3} \mathrm{CoC}_{4}$ on a macroscopic level: They render the two possible twin domains in $\mathrm{LT}-\mathrm{Sc}_{3} \mathrm{CoC}_{4}$ nearly indistinguishable and set the stage for the emergence of detwinned, single-domain crystals above $1.9 \mathrm{GPa}$. Thus a continuous superconducting sample volume may only be realized after the disappearance of twin domain walls from pressurized $\mathrm{Sc}_{3} \mathrm{CoC}_{4}$. Such a barrier function of twin domain walls for superconducting currents has been investigated recently by Song et al. [98] for FeSe.

We finally note that our results point to the simultaneous existence of volume superconductivity and a Peierls-type distorted phase at elevated pressures. There seems to be no pressure-adjustable competition between periodic structural distortion and superconductivity like in many other structurally low-dimensional materials $[15-17,29,87,88]$. Subtle pressure-induced modifications of the atom arrangement in the distorted phase might already suffice to reconcile both phenomena in $\mathrm{Sc}_{3} \mathrm{CoC}_{4}$.

\section{ACKNOWLEDGMENT}

We thank the group of Prof. Ch. Kuntscher for their continuous support in high-pressure studies. 
[1] T. Kim, K. Cho, E. Lee, S. Lee, J. Chae, J. Kim, D. Kim, J. Kwon, G. Amaratunga, S. Lee, B. Choi, Y. Kuk, J. Kim, and K. Kim, Nat. Photonics 5, 176 (2011).

[2] P. Patel, IEEE Spectrum 49, 14 (2012).

[3] R. Li, L. Petit, D. Franke, J. Dehollain, J. Helsen, M. Steudtner, N. Thomas, Z. Yoscovits, K. Singh, S. Wehner, L. Vandersypen, J. Clarke, and M. Veldhorst, Sci. Adv. 4, eaar3960 (2018).

[4] N. Dasgupta, J. Sun, C. Liu, S. Brittman, S. Andrews, J. Lim, H. Gao, R. Yan, and P. Yang, Adv. Mater. (Weinheim) 26, 2137 (2014).

[5] F. Schwierz, Nat. Nanotechnol. 5, 487 (2010).

[6] G. Hills, C. Lau, A. Wright, S. Fuller, M. Bishop, T. Srimani, P. Kanhaiya, R. Ho, A. Amer, Y. Stein, D. Murphy, Arvind, A. Chandrakasan, and M. Shulaker, Nature (London) 572, 595 (2019).

[7] F. Ando, Y. Miyasaka, T. Li, J. Ishizuka, T. Arakawa, Y. Shiota, T. Moriyama, Y. Yanase, and T. Ono, Nature (London) 584, 373 (2020).

[8] W. Little, Phys. Rev. 134, A1416 (1964).

[9] W. Little, Sci. Am. 212, 21 (1965).

[10] S. Pyon, K. Kudo, and M. Nohara, J. Phys. Soc. Jpn. 81, 053701 (2012).

[11] J. J. Yang, Y. J. Choi, Y. S. Oh, A. Hogan, Y. Horibe, K. Kim, B. I. Min, and S.-W. Cheong, Phys. Rev. Lett. 108, 116402 (2012).

[12] A. Kiswandhi, J. S. Brooks, H. B. Cao, J. Q. Yan, D. Mandrus, Z. Jiang, and H. D. Zhou, Phys. Rev. B 87, 121107(R) (2013).

[13] J. Hodeau, M. Marezio, C. Roucau, R. Ayroles, A. Meerschaut, J. Rouxel, and P. Monceau, J. Phys. C: Solid State Phys. 11, 4117 (1978).

[14] M. Regueiro, J.-M. Mignot, and D. Castello, EPL 18, 53 (1992).

[15] K. Cho, M. Kończykowski, S. Teknowijoyo, M. Tanatar, J. Guss, P. Gartin, J. Wilde, A. Kreyssig, R. McQueeney, A. Goldman, V. Mishra, P. Hirschfeld, and R. Prozorov, Nat. Commun. 9, 2796 (2018).

[16] J. Chang, E. Blackburn, A. Holmes, N. Christensen, J. Larsen, J. Mesot, R. Liang, D. Bonn, W. Hardy, A. Watenphul, M. v. Zimmermann, E. Forgan, and S. Hayden, Nat. Phys. 8, 871 (2012).

[17] A. Chikina, A. Fedorov, D. Bhoi, V. Voroshnin, E. Haubold, Y. Kushnirenko, K. Kim, and S. Borisenko, npj Quantum Mater. 5, 22 (2020).

[18] W. Knafo, F. Duc, F. Bourdarot, K. Kuwahara, H. Nojiri, D. Aoki, J. Billette, P. Frings, X. Tonon, E. Lelièvre-Berna, J. Flouquet, and L.-P. Regnault, Nat. Commun. 7, 13075 (2016).

[19] A. V. Chubukov, D. V. Efremov, and I. Eremin, Phys. Rev. B 78, 134512 (2008).

[20] P. Dai, J. Hu, and E. Dagotto, Nat. Phys. 8, 709 (2012).

[21] A. Meerschaut and J. Rouxel, J. Less-Common Met. 39, 197 (1975).

[22] L. Yang, Y. Tao, J. Liu, C. Liu, Q. Zhang, M. Akter, Y. Zhao, T. Xu, Y. Xu, Z. Mao, Y. Chen, and D. Li, Nano Lett. 19, 415 (2019).

[23] K. Novoselov, A. Geim, S. Morozov, D. Jiang, Y. Zhang, S. Dubonos, I. Grigorieva, and A. Firsov, Science 306, 666 (2004).

[24] Y. Cao, V. Fatemi, S. Fang, K. Watanabe, T. Taniguchi, E. Kaxiras, and P. Jarillo-Herrero, Nature (London) 556, 43 (2018).

[25] P. Williams, G. Parry, and C. Scrub, Philos. Mag. 29, 695 (1974).
[26] S.-H. Lee, J. S. Goh, and D. Cho, Phys. Rev. Lett. 122, 106404 (2019).

[27] H. Hughes, J. Phys. C: Solid State Phys. 10, L319 (1977).

[28] G.-J. Shu, Y. Zhou, M.-Y. Kao, C. Klingshirn, M. Huang, Y.-L. Huang, Y. Liang, W. Kuo, and S.-C. Liou, Appl. Phys. Lett. 114, 202103 (2019).

[29] H. Oike, M. Kamitani, Y. Tokura, and F. Kagawa, Sci. Adv. 4, eaau3489 (2018).

[30] G. Eickerling, C. Hauf, E.-W. Scheidt, L. Reichardt, C. Schneider, A. Muñoz, S. Lopez-Moreno, A. H. Romero, F. Porcher, G. André, R. Pöttgen, and W. Scherer, Z. Anorg. Allg. Chem. 639, 1985 (2013).

[31] B. Rohrmoser, G. Eickerling, M. Presnitz, W. Scherer, V. Eyert, R.-D. Hoffmann, U. Rodewald, C. Vogt, and R. Pöttgen, J. Am. Chem. Soc. 129, 9356 (2007).

[32] W. Jeitschko, M. Gerss, R.-D. Hoffmann, and S. Lee, J. LessCommon Met. 156, 397 (1989).

[33] A. Tsokol', O. Bodak, and E. Marusin, Sov. Phys. Crystallogr. 31, 466 (1986).

[34] W. Scherer, C. Hauf, M. Presnitz, E.-W. Scheidt, G. Eickerling, V. Eyert, R.-D. Hoffmann, U. C. Rodewald, A. Hammerschmidt, C. Vogt, and R. Pöttgen, Angew. Chem. Int. Ed. 49, 1578 (2010).

[35] E.-W. Scheidt, C. Hauf, F. Reiner, G. Eickerling, and W. Scherer, J. Phys.: Conf. Ser. 273, 012083 (2011).

[36] W. Scherer, G. Eickerling, C. Hauf, M. Presnitz, E.-W. Scheidt, V. Eyert, and R. Pöttgen, in Modern Charge-Density Analysis, 1st ed., edited by C. Gatti and P. Macchi (Springer, Dordrecht, 2012), Chap. 10, pp. 359-385.

[37] M. He, C. Wong, D. Shi, P. Tse, E.-W. Scheidt, G. Eickerling, W. Scherer, P. Sheng, and R. Lortz, J. Phys.: Condens. Matter 27, 075702 (2015).

[38] J. Langmann, C. Haas, E. Wenger, D. Schaniel, W. Scherer, and G. Eickerling, Phys. Rev. B 102, 094109 (2020).

[39] E. Wang, X. Zhu, and H.-W. Wen, EPL 115, 27007 (2016).

[40] C. Vogt, R.-D. Hoffmann, U. Rodewald, G. Eickerling, M. Presnitz, V. Eyert, W. Scherer, and R. Pöttgen, Inorg. Chem. 48, 6436 (2009).

[41] A. Jesche and P. Canfield, Philos. Mag. 94, 2372 (2014).

[42] C. Haas, Strukturelle und physikalische Charakterisierungen niederdimensionaler Metallborocarbide und Metallcarbide, Ph.D. thesis, University of Augsburg, 2019.

[43] See Supplemental Material at http://link.aps.org/supplemental/ 10.1103/PhysRevB.103.184101 for information on synthesis and properties of the investigated samples, details of the magnetization, resistivity and $\mathrm{x}$-ray diffraction experiments under ambient and high-pressure conditions, phonon dispersion relations under uniaxial strain, and remarks on the analysis of the experimental data.

[44] N. Tateiwa, Y. Haga, T. Matsuda, and Z. Fisk, J. Phys.: Conf. Ser. 500, 142032 (2014).

[45] N. Tateiwa, Y. Haga, T. Matsuda, Z. Fisk, S. Ikeda, and H. Kobayashi, Rev. Sci. Instrum. 84, 046105 (2013).

[46] N. Tateiwa, Y. Haga, T. Matsuda, and Z. Fisk, Rev. Sci. Instrum. 83, 053906 (2012).

[47] N. Tateiwa, Y. Haga, Z. Fisk, and Y. Ōnuki, Rev. Sci. Instrum. 82, 053906 (2011).

[48] T. Kobayashi, H. Hidaka, H. Kotegawa, K. Fujiwara, and M. Eremets, Rev. Sci. Instrum. 78, 023909 (2007). 
[49] K. Yokogawa, K. Murata, H. Yoshino, and S. Aoyama, Jpn. J. Appl. Phys. 46, 3636 (2007).

[50] K. Murata, K. Yokogawa, H. Yoshino, S. Klotz, P. Munsch, A. Irizawa, M. Nishiyama, K. Iizuka, T. Nanba, T. Okada, Y. Shiraga, and S. Aoyama, Rev. Sci. Instrum. 79, 085101 (2008).

[51] A. Eiling and J. Schilling, J. Phys. F: Met. Phys. 11, 623 (1981).

[52] B. Bireckoven and J. Wittig, J. Phys. E: Sci. Instrum. 21, 841 (1988).

[53] M. Nicklas, in Strongly Correlated Systems: Experimental Techniques, edited by A. Avella and F. Mancini (Springer, Berlin, 2015), Chap. 6, pp. 173-204.

[54] D. Schmitz, Experimentelle Hochdruckstudien an metallorganischen Modellsystemen mit aktivierten Kohlenstoff-Wasserstoff oder Silizium-Wasserstoff Bindungen, Ph.D. thesis, University of Augsburg, 2018.

[55] A. Le Bail, H. Duroy, and J. Fourquet, Mater. Res. Bull. 23, 447 (1988).

[56] J.-F. Bérar and P. Lelann, J. Appl. Crystallogr. 24, 1 (1991).

[57] V. Petříček, M. Dušek, and L. Palatinus, Z. Kristallogr. 229, 345 (2014).

[58] P. Willmott, D. Meister, S. Leake, M. Lange, A. Bergamaschi, M. Böge, M. Calvi, C. Cancellieri, N. Casati, A. Cervellino, Q. Chen, C. David, U. Flechsig, F. Gozzo, B. Henrich, S. JäggiSpielmann, B. Jakob, I. Kalichava, P. Karvinen, J. Krempasky et al., J. Synchrotron Radiat. 20, 667 (2013).

[59] M. Fisch, A. Lanza, P. Macchi, and N. Casati, J. Appl. Crystallogr. 48, 1956 (2015).

[60] A. Bergamaschi, A. Cervellino, R. Dinapoli, F. Gozzo, B. Henrich, I. Johnson, P. Kraft, A. Mozzanica, B. Schmitt, and X. Shi, J. Synchrotron Radiat. 17, 653 (2010).

[61] G. Piermarini, S. Block, and J. Barnett, J. Appl. Phys. 44, 5377 (1973).

[62] R. Angel, D. Allan, R. Miletich, and L. Finger, J. Appl. Crystallogr. 30, 461 (1997).

[63] D. Graf, R. Stillwell, K. Purcell, and S. Tozer, High Pressure Res. 31, 533 (2011).

[64] R. Boehler and K. De Hantsetters, High Pressure Res. 24, 391 (2004).

[65] K. Murata and S. Aoki, Rev. High Pressure Sci. Technol. 26, 3 (2016).

[66] G. Piermarini, S. Block, J. Barnett, and R. Forman, J. Appl. Phys. 46, 2774 (1975).

[67] A. Dewaele, M. Torrent, P. Loubeyre, and M. Mezouar, Phys. Rev. B 78, 104102 (2008).

[68] I. Kantor, Fluorescense pressure calculation and thermocouple tools, accessed December 14, 2020, http://kantor.50webs.com/ ruby.htm.

[69] R. Boehler, Rev. Sci. Instrum. 77, 115103 (2006).

[70] A. Duisenberg, J. Appl. Crystallogr. 25, 92 (1992).

[71] A. Duisenberg, L. Kroon-Batenburg, and A. Schreurs, J. Appl. Crystallogr. 36, 220 (2003).

[72] L. Krause, R. Herbst-Irmer, G. Sheldrick, and D. Stalke, J. Appl. Crystallogr. 48, 3 (2015).

[73] Joint CCDC-FIZ Karlsruhe online deposition service, https: //www.ccdc.cam.ac.uk/structures/?; access by quoting the deposition numbers CSD-2060775, CSD-2060779, and CSD2060780.

[74] G. Kresse and J. Furthmüller, Comput. Mater. Sci. 6, 15 (1996).

[75] G. Kresse and J. Furthmüller, Phys. Rev. B 54, 11169 (1996).

[76] G. Kresse and J. Hafner, Phys. Rev. B 49, 14251 (1994).
[77] G. Kresse and J. Hafner, Phys. Rev. B 47, 558 (1993).

[78] J. P. Perdew, K. Burke, and M. Ernzerhof, Phys. Rev. Lett. 77, 3865 (1996).

[79] R. Elmer, M. Berg, L. Carlen, B. Jakobsson, B. Noren, A. Oskarsson, G. Ericsson, J. Julien, T. F. Thorsteinsen, M. Guttormsen, G. Lovhoiden, V. Bellini, E. Grosse, C. Muntz, P. Senger, and L. Westerberg, Phys. Rev. Lett. 78, 1396 (1997).

[80] A. Togo and I. Tanaka, Scr. Mater. 108, 1 (2015).

[81] F. Brown, in Structural Phase Transitions in Layered Transition Metal Compounds, edited by K. Motizuki, Physics and Chemistry of Materials with Low-Dimensional Structures Vol. 8 (Springer, Dordrecht, 1986), Chap. 4, pp. 267-292.

[82] Y. Joe, X. Chen, P. Ghaemi, K. Finkelstein, G. de La Peña, Y. Gan, J. Lee, S. Yuan, J. Geck, G. MacDougall, T. Chiang, S. Cooper, E. Fradkin, and P. Abbamonte, Nat. Phys. 10, 421 (2014).

[83] N. Wakabayashi, H. Smith, K. Woo, and F. Brown, Solid State Commun. 28, 923 (1978).

[84] D. Moncton, J. Axe, and F. DiSalvo, Phys. Rev. Lett. 34, 734 (1975).

[85] D. Moncton, J. Axe, and F. DiSalvo, Phys. Rev. B 16, 801 (1977).

[86] A. Kusmartseva, B. Sipos, H. Berger, L. Forro, and E. Tutiš, Phys. Rev. Lett. 103, 236401 (2009).

[87] D. C. Freitas, P. Rodière, M. R. Osorio, E. Navarro-Moratalla, N. M. Nemes, V. G. Tissen, L. Cario, E. Coronado, M. GarcíaHernández, S. Vieira, M. Núñez-Regueiro, and H. Suderow, Phys. Rev. B 93, 184512 (2016).

[88] C. Berthier, P. Molinié, and D. Jérome, Solid State Commun. 18, 1393 (1976).

[89] Y. Feng, J. Wang, R. Jaramillo, J. Van Wezel, S. Haravifard, G. Srajer, Y. Liu, Z.-A. Xu, P. Littlewood, and T. Rosenbaum, Proc. Natl. Acad. Sci. U. S. A. 109, 7224 (2012).

[90] C. D. Haas, G. Eickerling, E.-W. Scheidt, D. Schmitz, J. Langmann, J. Lyu, J. Shen, R. Lortz, D. Eklöf, J. G. Schiffmann, L. van Wüllen, A. Jesche, and W. Scherer, arXiv:1712.05649v3.

[91] C. Zhang, J. Tse, K. Tanaka, and H.-Q. Lin, EPL 100, 67003 (2012).

[92] This twinning process is due to a $t 2$ step followed by an $i 2$ step in the symmetry reduction (translationengleiche and isomorphic group-subgroup relationship, respectively) from the space group Immm of the HT phase structure to the space group $C 2 / m$ of the LT phase structure; see Refs. [30] and [40].

[93] Conrotatory displacements make no contribution to the intensities of the superstructure reflections and only a minor contribution to the intensities of the main reflections; that is, even a hypothetical rotation by $15^{\circ}$ changes the average main reflection intensity by less than $1 \%$. A more detailed discussion is available in the Supplemental Material [43].

[94] Only minor nonhydrostaticity can be expected below the hydrostatic limit of the employed pressure-transmitting medium Daphne 7575 at 3.9-4 GPa ( $T=298 \mathrm{~K}$; see Ref. [65]). Exemplary room-temperature fluorescence measurements on different ruby spheres revealed no pressure gradient inside the pressure chamber at $4 \mathrm{GPa}$. Also a solidification of the pressure medium upon cooling should not induce significant nonhydrostaticity, as demonstrated in Ref. [99]. Consistently, we observed anisotropic broadening of reflection profiles only for x-ray diffraction data collected at pressures well above $4 \mathrm{GPa}$. 
[95] L. Zhang, C. Fehse, H. Eckert, C. Vogt, R.-D. Hoffmann, and R. Pöttgen, Solid State Sci. 9, 699 (2007).

[96] C. Vogt, R.-D. Hoffmann, and R. Pöttgen, Solid State Sci. 7, 1003 (2005).

[97] The fact that the calculations do not predict a preferential phonon frequency reduction at $T$ may be related to shortcomings of the standard generalized gradient approximation (GGA) functional employed within this study to properly resolve the very flat energy surface region separating the HT and LT phases of $\mathrm{Sc}_{3} \mathrm{CoC}_{4}$.

[98] C.-L. Song, Y.-L. Wang, Y.-P. Jiang, L. Wang, K. He, X. Chen, J. E. Hoffman, X.-C. Ma, and Q.-K. Xue, Phys. Rev. Lett. 109, 137004 (2012).

[99] D. Staško, J. Prchal, M. Klicpera, S. Aoki, and K. Murata, High. Pressure Res. 40, 525 (2020). 